\title{
Editorial
}

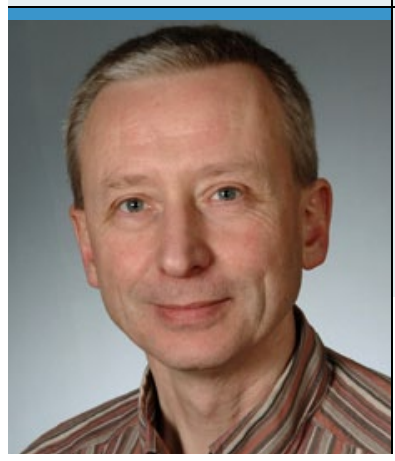

„Ein großer Teil des gesellschaftlichen Prozesses

auf dem Weg zu kindgerechten Schulen liegt noch vor uns."

Dr. med. Wolfgang Broxtermann,

Ärztlicher Leiter des Kinderzentrums Pelzerhaken, Neustadt in Holstein

\section{Kinderärzte sollen Katalysatoren sein}

Liebe Kolleginnen und Kollegen,

noch nie wurde in Deutschland so intensiv, so professionell und so breit gesellschaftlich gestreut über Kinder, Familie und Schule nachgedacht und diskutiert. Es ist eine überaus positive Entwicklung, dass die Politik beginnt, Kinder ernst zu nehmen und zu verstehen.

Kinder- und Jugendärzte sind Katalysatoren auf dem Weg zum „gleichwürdigen Umgang“" (Jesper Juul) von Eltern und ihren Kindern und für den Schritt von der Erziehung zur Beziehung.

Jedes Kind befindet sich in einer individuellen Situation und muss separat gesehen und gefördert werden. Die Stärken/Schwächen-Profile der Schulkinder sind zumeist unbekannt und werden noch an keiner Stelle systematisch untersucht, bevor schulische Anforderungen gestellt werden. Der zeitliche Rahmen von Schuluntersuchungen ist so kurz, dass keine relevanten Aussagen über das Leistungsprofil möglich sind. Noch immer wenden Schulkinder den größten Teil ihrer Energie für Fächer auf, in denen sie keine Begabung haben.

Erst im Erwachsenenalter wird es den jungen Menschen freigestellt, sich nach ihren Neigungen und Fähigkeiten zu entscheiden. Ein großer Teil des gesellschaftlichen Prozesses auf dem Weg zu kindgerechten Schulen und der Akzeptanz eines individuellen Weges der Kinder ab ihrer Geburt liegt noch vor uns.
Von der Ernährung bis zu den neuen Medien, von der Krippenerziehung bis zum Absentismus, vom Autismus bis zur Inklusion, von ADHS bis zum Blick aus der Praxis und schließlich vom Gesundheitsbericht der Schulanfänger bis zum Interview mit Prof. Klaus Hurrelmann spannt sich der Bogen der hier zusammengefassten Arbeiten.

Aber auch wenn der Alltag in den Praxen und Sozialpädiatrischen Zentren häufig mühevoll erscheint und viele Rückschläge unseren Elan manchmal schwinden lassen, so zeigt sich doch beim Blick von außen und über die Zeit: Wir sind auf dem richtigen Weg!

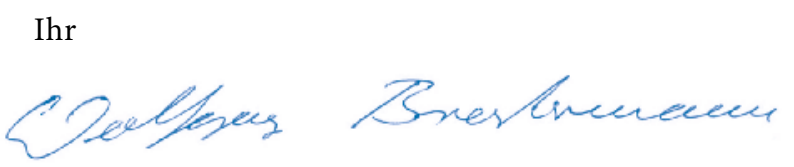

